\title{
Reformuler et justifier les propos dans les cercles de lecture après enseignement de l'oral : différences entre élèves et perception des enseignants
}

\author{
MANON HÉBERT, Université de Montréal
}

AMÉLIE GUAY, Université du Québec en Outaouais

LIZANNE LAFONTAINE, Université du Québec en Outaouais

\section{Résumé}

Dans sept classes (primaire-secondaire), des élèves ont effectué deux séries de cercles de lecture entre pairs (CLP) en cours d'année, soit avant et après des ateliers d'enseignement explicite de l'oral. Nous avons analysé les transcriptions d'un échantillon de 14 CLP puis retenu 18 élèves-cas. Nous présentons nos résultats au sujet des différences observées quant à leurs habiletés à reformuler, à justifier et à élaborer leurs propos, et aussi quant à leur opinion au sujet de leurs apprentissages et participation. Nous rapporterons également les commentaires des enseignants sur les intérêts et limites de cette approche intégrée pour le développement des habiletés en littératie.

\section{Introduction}

$\mathrm{Au}$ moment d'entreprendre cette recherche, l'enquête internationale du Programme international pour le suivi des acquis des élèves (PISA, 2001) avait révélé que $55 \%$ des adolescents québécois âgés de 15 ans ne pouvaient réussir des tâches de lecture complexes, comme interpréter les nuances de la langue et évaluer un texte. Cela rejoint également les propos de McCraken et Murray (2009) qui affirment qu'entre 20 et $40 \%$ des élèves canadiens n'auraient pas les compétences suffisantes en littératie pour fonctionner en société. Sans parler des récents résultats du Programme pour l'évaluation internationale des compétences des adultes (2012) montrant que plus de la moitié de la population québécoise de 16 à 65 ans possède des compétences insuffisantes en littératie pour participer activement au monde actuel (Statistique Canada, 2013). C'est donc dire que l'enseignement des compétences en littératie mérite encore d'être amélioré, notamment, par le développement d'approches visant à intégrer la lecture, l'écriture et l'oral (Hébert, $2010: 6$ ).

Pour contribuer à palier ce problème, nous avons mené une recherche qualitative de 2009 à $2014^{1}$ auprès de quatre classes de troisième cycle du primaire et de trois classes

\footnotetext{
${ }^{1}$ Hébert, M. et Lafontaine L. (2009-2014), L'enseignement-évaluation intégré de l'oral réflexif dans les situations d'apprentissage par les pairs en lecture: quels caractéristiques, indices de progressiondifférenciation et difficultés d'enseignement (primaire/secondaire). Projet subventionné par le FRQSC (Fonds de recherche du Québec - Société et culture), dans le cadre du concours « Actions concertées sur la persévérance scolaire $»$.
} 
de troisième secondaire dans les régions de Montréal (milieu urbain) et des Laurentides (milieu semi-rural) dans lesquelles les élèves ont fait des cercles de lecture entre pairs (CLP) à partir du roman de science-fiction Le Passeur, de Lois Lowry. Afin de mieux comprendre comment enseigner-évaluer l'oral en situation d'interaction et d'intégration des compétences (mieux discuter pour mieux lire), nous avons retenu les trois objectifs suivants: 1) dégager quelles sont les caractéristiques de l'objet à enseigner en oral (soit un oral spontané et réflexif lié aux situations d'apprentissage en lecture par les pairs) pour développer des outils d'enseignement/évaluation appropriés; 2) expérimenter en classe deux modèles innovants d'enseignement explicite et intégrés, soit le modèle transactionnel en lecture de Hébert (2003) et le modèle didactique de la production orale de Lafontaine (2001), cela en examinant où se situent les difficultés des enseignants dans ce contexte; 3) analyser les productions d'élèves à l'oral afin de dégager des indices de progression.

Dans cet article, nous présentons la problématique de l'enseignement de l'oral réflexif et situons les courants théoriques liés à l'enseignement de l'oral qui sont les assises de notre recherche. Puis, nous exposons notre méthodologie ainsi que notre démarche d'enseignement de l'oral (modèle didactique de la production orale de Lafontaine, 2001) ainsi que nos dispositifs (ateliers portant sur la reformulation et la justification, relatifs à l'objectif 2). Enfin, nous présenterons les résultats qui ont trait aux différences observées dans l'usage de la reformulation et de la justification des 18 élèvescas retenus (objectif 3 ) et nous relatons les perceptions des enseignants et des élèves par rapport aux apprentissages effectués dans ce contexte.

\section{1- Problématique}

Dans une enquête réalisée par le ministère de l'Éducation, du Loisir et du Sport en 2007 (MÉLS : 24), $70 \%$ des 2000 enseignants du primaire interrogés trouvaient difficile d'enseigner-évaluer la compétence Apprécier des æuvres littéraires, ajouté au fait que la compétence Communiquer oralement constituait la deuxième compétence la moins travaillée en français. Et selon plusieurs recherches, les enseignants de français du secondaire ne savent pas comment enseigner ni évaluer l'oral (Dolz et Schneuwly, 1998; Lafontaine, 2001; Lafontaine et Préfontaine, 2007; Sénéchal, 2012; Chartrand et Lord, 2013). En classe de français, l'oral devrait être enseigné-évalué en tant qu'objet ou compétence disciplinaire et être intégré aux autres apprentissages, selon ce qui notamment est recommandé dans le programme d'études québécois du primaire (ministère de 1'Éducation du Québec, 2001 : 91). Cependant, cette intégration souhaitée constitue une grande difficulté en classe inclusive et pour laquelle la recherche n'a pas encore fourni beaucoup de propositions concrètes. L'oral en tant qu'objet et médium d'apprentissage est très peu enseigné dans les classes de français et plusieurs recherches en recommandent un enseignement explicite (Dolz et Schneuwly, 1997; Lafontaine et Messier, 2009). Pour ce faire, et selon l'approche intégrée préconisée par Nonnon (1999 : 8), il faudrait parvenir à formaliser les différentes pratiques de verbalisation de chaque tâche scolaire, en fonction de la spécificité du contenu discuté et des apprentissages visés.

Dans la même foulée, on en sait très peu sur la particularité de la verbalisation dans les CLP, un genre scolaire informel où conversation, dialogue et controverse se 
mêlent pendant la coconstruction des interprétations en lecture (Jacques, 1988). Il s'agit d'un type d'oral spontané et interactif dans une situation mettant en jeu plusieurs compétences ainsi que d'un oral «pour apprendre » et non pas «à apprendre » parce qu'il sert avant tout à construire les savoirs en interaction. Ce genre de tâche conduit ainsi les élèves à utiliser un oral dit "réflexif» selon Jaubert et Rebière (2002). Le déroulement des CLP est souple: quatre à six élèves hétérogènes discutent pendant 30 à 45 minutes des stratégies de lecture qu'ils ont utilisées pendant leur lecture d'un même roman (séparé en tranches de lecture). Deux seuls rôles sont prédéfinis : animateur (s'assurer que chaque élève prend la parole pour présenter sa stratégie) et secrétaire (prendre en note les stratégies discutées par chaque élève). À tour de rôle, chaque élève prend la parole de la manière suivante : amener le sujet en situant et en résumant le passage à discuter, citation à l'appui; poser le sujet en identifiant la stratégie de lecture utilisée; justifier et élaborer son propos à l'aide des autres élèves (Hébert, 2003). À la suite du CLP, chacun rédige de un à trois commentaires dans son journal de lecture en suivant la même structure (80 à 150 mots selon l'âge). Le CLP favorise ainsi l'intégration de la lecture, de l'oral réflexif et de l'écriture.

Cela étant dit, comment enseigner-évaluer les habiletés orales exigées dans un genre de tâche réflexive en situation d'interaction entre pairs comme le sont les CLP? Quelles habiletés seraient prioritaires à enseigner et comment cela varierait-il selon l'âge? Ce manque de connaissances fines sur l'objet à enseigner amène les enseignants à trop souvent utiliser les CLP de manière indifférenciée : ils savent peu distinguer les notions spécifiques à l'oral et en lecture qu'il conviendrait d'enseigner et, selon Lafontaine (2001), encore moins les évaluer et en penser la progression. En lecture, il faut par ailleurs accepter l'idée que «l'intérêt (ou la motivation) des élèves (et des enseignants) ne réside pas seulement dans les contenus (les textes, l'histoire, la nouveauté, les connaissances culturelles): l'apprentissage et la prise de conscience des stratégies efficaces [...], le développement d'un sentiment de contrôle ainsi pris sur leur activité sont aussi des sources de motivation extrêmement puissantes " (Cèbe, Goigoux et Thomazet, 2003: 6). Les recherches en psychologie cognitive ont en effet montré l'importance de l'enseignement des stratégies de lecture puisque, en l'absence de connaissances adéquates dans un domaine donné (par exemple en littérature), les stratégies de lecture que possède un individu constituent une variable fondamentale du processus d'apprentissage et deviennent la ressource privilégiée du lecteur pour réussir sa tâche et en retirer un certain sentiment de réussite (Deschênes, 1991: 30). Dans le cas des élèves en difficulté, des activités interactives comme les CLP sont jugées parmi les pratiques d'enseignement très efficaces. On connaît l'importance de la verbalisation des processus d'apprentissage sur un plan cognitif et du travail entre pairs pour la motivation des élèves (Hébert, 2003, 2007). Le modelage des pairs leur rendrait le processus de lecture plus transparent, ils y développeraient une vision plus positive d'eux-mêmes en tant que lecteurs/orateurs et aussi un sentiment plus grand d'autonomie et d'appartenance (O’Brien, 2006; Blum, Lipsett et Yocom, 2002).

\section{2- Cadre théorique}

Nous présentons le cadre socio-interactionniste sur lequel s'appuie notre projet quant aux indicateurs que nous avons retenus pour l'enseignement et l'analyse à propos 
de l'oral réflexif. Nous nous concentrons surtout sur les deux qui font partie intégrante des CLP, à savoir la reformulation, sur un plan langagier, et la justification, sur un plan discursif.

\subsection{Le cadre socio-interactionniste}

La perspective socio-interactionniste sur l'acquisition des langues attribue à l'interaction sociale un rôle central dans les processus acquisitionnels (Pekarek-Doehler, De Pietro, Fasel Lauzon et Pochon-Berger, 2008). Cette perspective favorise la compréhension de la compétence d'interaction et d'organisation du discours en interaction (Berger, 2008; Fasel Lauzon, Pekarek-Doehler et Pochon-Berger, 2009). En effet, cette compétence langagière intègre diverses conduites discursives liées les unes aux autres (par exemple, prendre la parole, justifier, reformuler) comme en témoigne l'utilisation qu'en font les élèves dans les CLP. La perspective socio-interactionniste permet de mieux comprendre la spécificité des communautés discursives ou d'apprentissage dans chaque matière (Bernié, 2003; Bouchard, 2004). Par exemple, il faut mieux analyser le contrat de communication attendu dans la classe de littérature lorsqu'on demande à l'élève de "justifier » ses propos, une conduite discursive qui doit en plus s'opérer en situation d'interaction entre pairs dans le cadre des CLP.

Les interactions orales dans les CLP visent d'abord à permettre aux élèves de construire des connaissances en lecture. L'oral est donc ici un médium d'enseignement parce qu'il est le véhicule utilisé pour s'exprimer dans ces activités en sous-groupes où les élèves discutent d'un roman commun, prennent en compte le destinataire, font preuve d'écoute active, reformulent pour s'assurer d'avoir bien compris ce que l'autre a dit, justifient, etc. (Hébert, 2003). Il s'agit ainsi d'un oral dit « réflexif » (Crinon, 2002; Chabanne et Bucheton, 2002). Cependant, comme l'utilisation de conduites discursives propres à cette situation de communication est alors attendue, comme expliquer et justifier son propos (Dreyfus et Cellier, 2000; Lafontaine et Blain, 2007), nous avons fait l'hypothèse que ce type d'oral réflexif peut aussi devenir objet d'enseignement lorsqu'il est enseigné de manière explicite afin d'amener les élèves à prendre en charge de façon consciente ces aspects de leur communication orale. Selon Lusetti (2004), travailler l'oral réflexif en tant qu'objet d'enseignement dans la classe permet aux enseignants de proposer des objets d'apprentissage de l'oral concrets (ici la reformulation et la justification), d'inculquer des stratégies d'apprentissage aux élèves et d'ajouter du plaisir à la tâche orale.

\subsection{Indicateurs de l'oral réflexif sur les plans discursif, interactionnel et langagier}

Lors de l'analyse de données secondaires issues d'une recherche antérieure et qui nous a permis d'atteindre notre objectif $1^{2}$, nous avons cherché à identifier quels indicateurs mériteraient un enseignement spécifique parmi les indicateurs dégagés au départ par Hébert (2003: 351). Nous avons retenu deux dimensions et neuf indicateurs : dimension interactionnelle (indicateurs " prise en compte de l'autre », " aide aux pairs » et « reprise/reformulation ») et dimension discursivo-langagière (indicateurs "procédés

\footnotetext{
${ }^{2}$ Hébert, M. (2005-2008), Étude longitudinale des indices de progression et des modalités de co-évaluation dans les cercles littéraires entre pairs (primaire/secondaire), projet subventionné par le FQRSC (Fonds québécois de la recherche sur la société et la culture).
} 
discursifs ", dont la justification, les marqueurs de relation, la prise en charge de son discours et la généralisation) (Hébert et Lafontaine, 2012b). Deux indicateurs dans le contexte des CLP nous sont apparus incontournables, soit la justification et la reformulation.

2.2.1 La justification. Les analyses que nous avons effectuées pendant la première année ont mis au jour que les conduites discursives dominantes dans les CLP sont d'abord de l'ordre de la justification (Hébert et Lafontaine, 2012a). Effectivement, selon Fasel Lauzon (2009 : 27), le discours justificatif porte sur des croyances et est subjectif, ce qui est en lien avec le contenu discuté dans nos CLP, alors que « le discours explicatif porte sur des faits établis et est objectif, c'est-à-dire dénué de polémique ». La structure de l'explication comprendrait trois phases selon Fasel Lauzon (2009 : 30) : une ouverture - problématisation de l'objet à expliquer; un noyau - résolution du problème; une clôture - marque de réception ou de ratification de l'explication. Nous avons postulé qu'il pouvait aussi en être de même pour la structure de la justification comme nous le verrons dans les résultats. Par ailleurs, dans le cadre de cette tâche scolaire qui portait sur l'appréciation des textes littéraires, nous avons aussi considéré le degré d'élaboration de la justification (jugé selon des critères de cohésion, pertinence, justesse et solidité du propos) (Hébert, 2003).

2.2.2 La reformulation. Plusieurs didacticiens de l'oral ont étudié le rôle de la reformulation sur les plans langagier, discursif et interactionnel. Par exemple, pour ce qui est de la dimension langagière, Le Cunff (2004) précise que la reformulation est une forte entrée dans l'oral en situation d'interaction au primaire puisqu'elle suppose un travail d'écoute et de synthèse. Lafontaine et Blain (2007) ont montré quant à elles que, dans les groupes de révision rédactionnelle entre pairs, les élèves plus forts progressaient dans les habiletés de reformulation et que les élèves plus faibles s'amélioraient en écoute.

Sur un plan discursif, Garcia-Debanc et Volteau (2007 : 327) affirment, en citant Gülich et Kotschi (1987), le lien étroit existant entre marqueurs de relation et reformulation puisque "la relation sémantique qui s'établit entre l'énoncé source et l'énoncé reformulateur se réalise par la présence d'un marqueur reformulatif ». Ces chercheuses précisent les différents rôles que la reformulation peut avoir selon les divers moments d'enseignement (pendant les consignes, en clôture, etc.). Jaubert (2005) a étudié les signes distinctifs des marqueurs et des reformulations liés aux processus d'abstraction, par exemple, les reformulations syntaxiques afin de réduire l'énoncé et les reformulations lexicales pour trouver le mot juste. De leur côté, Dreyfus et Cellier (2000) disent que les reformulations jouent un rôle très important dans l'élaboration de conduites explicatives comme celles que l'on observe dans les CLP, car elles font état d'un effort d'objectivation. Enfin, sur un plan interactionnel, De Pietro (1990: 22) définit la reformulation comme une méthode "à laquelle on recourt pour résoudre des difficultés (réelles ou présumées) de formulation et/ou d'intercompréhension ». La reformulation serait également une façon pour des locuteurs débutants en langue seconde et pour des élèves en difficulté d'entrer dans les tours de parole et de prendre leur place dans les conversations comme locuteurs compétents (Berger, 2008). 
Nous avons déjà rapporté, dans des articles en lien avec notre premier objectif de recherche (Hébert et Lafontaine, 2012ab), comment pouvaient se traduire les nombreuses fonctions et dimensions de la reformulation en situation d'interactions entre pairs à visée réflexive. La reformulation est une ressource utilisée à toutes les étapes de la justification dans les CLP : en ouverture, elle renvoie à la dimension langagière et cognitive, notamment par l'utilisation du discours rapporté pour citer le texte du roman; puis en noyau, pour le développement de la justification, la reformulation touche aux dimensions interactionnelle et cognitive par la prise en compte des propos de l'autre; et en clôture, la reformulation peut se rapporter à la synthèse des propos. Pour les présentes analyses, nous avons retenu que, dans un échange oral en interaction comme les CLP, le locuteur peut reformuler le propos des autres ou ses propres propos, ce que Garcia-Debanc et Volteau (2007 : 312) nomment l'hétéro-reformulation ou l'auto-reformulation, et ce, dans le but de répéter, de rectifier ou de synthétiser les propos.

\section{3- Méthodologie}

Dans cette section, nous décrivons 1) les participants; 2) le déroulement de l'année d'expérimentation en classe (qui correspond à l'objectif 2 de notre recherche); 3) les outils de collecte dont découlent les données analysées; et 4) les modes de traitement et d'analyse de ces données. Les précisions quant aux démarches et dispositifs d'enseignement adoptés en cours d'expérimentation (notamment les CLP et les étapes d'enseignement de l'oral) sont détaillées en annexe 1.

\subsection{Participants}

Le projet s'est déroulé dans quatre écoles : deux de Montréal, un milieu urbain à très forte concentration multiethnique, et deux dans la région des Laurentides, un milieu semi-rural essentiellement francophone. Quatre classes du primaire (trois de $6^{\mathrm{e}}$ année et une de $5^{\mathrm{e}}$ année, 10 à 12 ans) et trois classes de $3^{\mathrm{e}}$ secondaire (14-15 ans) ont été choisies sur le principe d'un échantillon de convenance $(n=170$ élèves), dans les classes d'enseignants qui avaient répondu à notre proposition et accepté de travailler en duo avec un collègue de leur école. De plus, un conseiller pédagogique par école $(\mathrm{n}=4)$ a été invité à participer au projet afin d'assurer un meilleur encadrement des enseignants. Dans chaque classe, nous avions cinq à six équipes de CLP (de 4-5 élèves) pour un total de 14 : sept en préexpérimentation et sept en expérimentation. De manière à pouvoir observer l'influence éventuelle de l'enseignement de l'oral dans ce contexte, nous avons choisi d'analyser le parcours de 18 élèves-cas (un élève fort, un moyen et un en difficulté dans chaque équipe en général) tirés de sept équipes (une par classe), soit 10,6 \% des élèves. Le niveau des élèves avait été documenté dès le début du projet, notamment à l'aide des résultats obtenus dans l'année en lecture, en écriture et en communication orale, et de l'avis des enseignants. Les critères de sélection des équipes étaient la présence des élèves à toutes les étapes du projet, la qualité des enregistrements et la constance des membres de l'équipe (qui devaient tous avoir participé à la pré-expérimentation et à l'expérimentation et aussi avoir signé le formulaire de consentement). 


\subsection{Déroulement}

L'année d'expérimentation en classe, liée à l'objectif 2 de la recherche, s'est déroulée en trois phases (voir figure 1). La phase de préexpérimentation à l'automne 2011, pour laquelle les enseignants ont reçu une formation d'une journée, visait à familiariser les élèves avec les CLP et le journal de lecture sur un roman choisi par l'enseignant (voir annexe 1). Au cours de cette phase, un enseignement explicite des stratégies de lecture, de quelques notions littéraires à l'aide d'extraits de textes et des attentes au sujet de l'écriture d'un journal axé sur l'utilisation des stratégies de lecture ${ }^{3}$ a été réalisé (Hébert 2003, 2004, 2006a, 2006b, 2008).
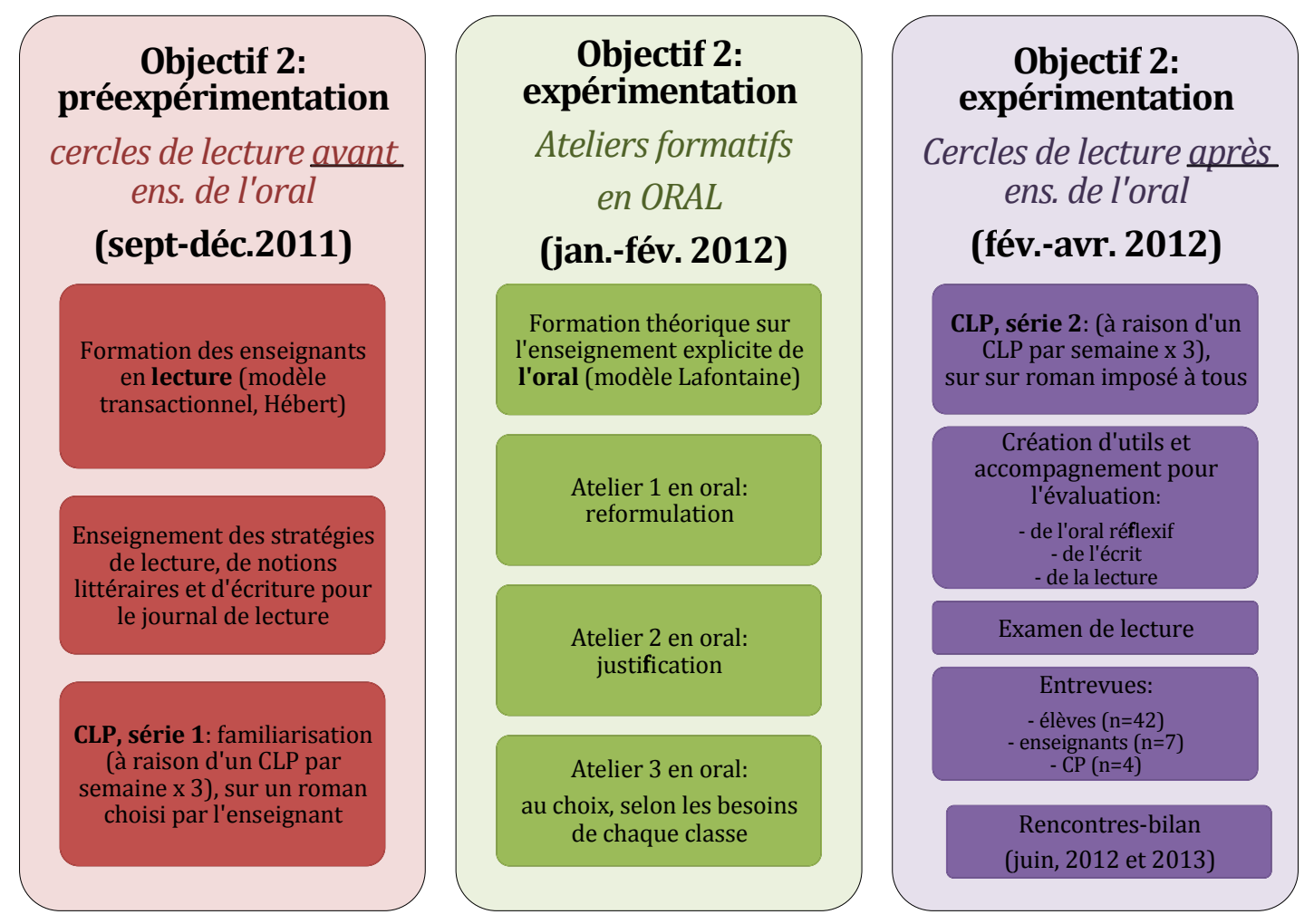

Figure 1. Phases de l'expérimentation

Pour la deuxième phase, les premiers CLP filmés ont été réutilisés à titre de productions initiales dans la phase d'enseignement de l'oral qui a eu lieu à l'hiver 2012, avant la phase d'expérimentation du printemps. Ainsi, en janvier, les enseignants ont été conviés à une deuxième journée de formation au modèle didactique de la production orale de Lafontaine (2001). Nous leur avons présenté la structure du modèle, les deux ateliers d'oral réflexif (sur la reformulation et sur la justification) que nous comptions expérimenter avec eux, puis nous les avons invités à préparer un troisième atelier en lien avec les besoins de leurs élèves. L'enseignement des deux premiers ateliers a été fait dans

\footnotetext{
${ }^{3}$ Par exemple: se questionner; faire des liens; interpréter; prédire; déduire; ressentir; juger, évaluer; revenir sur son opinion; visualiser, etc.
} 
chaque classe de février à mars 2012 par la cochercheuse, les enseignants n'étant pas encore à l'aise à ce sujet et préférant être observateurs. Ils ont toutefois enseigné leur troisième atelier, parfois en collaboration avec leur conseillère pédagogique. Toutes les séances d'enseignement ont été filmées et se sont étalées sur trois ou quatre semaines, à raison d'un atelier par semaine environ.

Ensuite, durant la troisième phase qui a eu lieu de mars à avril 2012, les élèves ont fait à nouveau trois CLP portant cette fois sur un même roman de science-fiction imposé à tous, puis des outils d'évaluation ont été développés et appliqués pour évaluer l'ensemble des activités en lecture (examen), en écriture (journal de lecture) et en oral (CLP).

\subsection{Outils de collecte de données}

À chacune des phases de l'expérimentation, diverses données contextuelles ont été recueillies et consignées pour mieux comprendre le cheminement de chaque élève-cas et saisir le contexte d'expérimentation du point de vue des enseignants et des chercheuses. Lors de la préexpérimentation en novembre-décembre, les résultats scolaires obtenus en lecture, en écriture et en oral qui avaient été colligés par les enseignants depuis le début de l'année ont été consultés, de même qu'un questionnaire que les élèves avaient complété pour se présenter sur un plan personnel (langues parlées, loisirs, motivation scolaire, comportement et perception de leur compétence en lecture, en écriture et en oral) et une lettre aux chercheuses qu'ils avaient rédigée pour décrire leurs habitudes de lecture. Ces outils ont servi à composer des équipes hétérogènes pour les CLP, puis à guider la sélection des cas en fin d'année. Pendant la première série de CLP, les élèves ont également réalisé des autoévaluations au sujet de leurs habiletés à participer et à élaborer leurs propos. Les enseignants ont quant à eux commenté les journaux de lecture de chaque élève.

Dans la phase d'enseignement de l'oral, les élèves ont été invités à compléter un portrait de locuteur où ils se sont situés comme locuteur (situations scolaires dans lesquelles l'oral était une expérience positive ou négative, etc.). Puis, dans un portfolio pour l'oral, ils ont colligé les différents référentiels et fiches synthèse en lien avec chacun des ateliers et ont constamment réfléchi à leurs apprentissages à ces sujets. Lors de la dernière série de CLP, ils ont à nouveau réalisé des autoévaluations. Les enseignants ont évalué leurs journaux de lecture et leurs cercles de lecture à l'aide de grilles d'évaluation construites par les chercheuses à cet effet et tous les élèves ont aussi fait un même examen de lecture sur le roman imposé.

Après les derniers CLP, des entretiens semi-dirigés d'une durée de 15 minutes sur les apprentissages faits ou non en oral ont été réalisées avec les 18 élèves-cas choisis (voir annexe 2). Des entretiens semi-dirigés d'une durée de 30 à 45 minutes ont aussi été réalisées avec les sept enseignants (en 2012 et 2013), en plus de bilans écrits (janvier et juin). Les enseignants ont par ailleurs rempli un journal de bord, de même que les conseillers pédagogiques et les deux chercheuses, lesquelles ont effectué de nombreuses observations in situ, notamment pendant tous les cours liés à l'application/expérimentation des modèles, tant lors des ateliers formatifs que lors des CLP. 


\subsection{Traitement et méthode d'analyse des données}

Dans une recherche de type qualitatif, la validité (vraisemblance des résultats) et la fiabilité (résultats dignes de confiance) sont des critères de scientificité importants. La triangulation entre les divers types de données est l'une des méthodes privilégiées pour assurer la validité et la fiabilité.

Pour l'analyse des indicateurs liés à l'utilisation de la reformulation et de la justification dans les CLP, les données proviennent essentiellement des transcriptions de CLP. Chaque équipe d'élèves retenue $(\mathrm{n}=7)$ a choisi un CLP à faire évaluer, qui a ensuite été transcrit et codé par les chercheuses et assistants afin d'évaluer les indices de progression après enseignement de l'oral, selon les principaux indicateurs reliés à ce qui avait été enseigné (reformulation, justification, degré d'élaboration). Nous avons transcrit et analysé au total 14 CLP (sept avant et sept après l'enseignement de l'oral). Trois élèves jugés forts, moyens et en difficulté selon l'enseignant ont été sélectionnés dans chaque classe, pour un total de 18 (neuf élèves-cas au primaire et neuf au secondaire). Les CLP ont été découpés en épisodes (groupe d'unités de sens se rapportant au même sujet de discussion autour d'une stratégie de lecture) $(\mathrm{n}=172)$ et en tours de parole (TP, $\mathrm{n}=4$ 800). Chaque TP des élèves a fait l'objet d'une analyse qualitative de contenu par catégories, liées entre autres aux éléments enseignés à l'oral (types de reformulation et de justification) et d'un contre-codage avec mesures de fidélité inter-juges.

Des codes ont été peu à peu déterminés pour chacun des principaux indicateurs relatifs : a) à l'utilisation des procédés linguistiques enseignés (types de reformulation ${ }^{4}$ ); au type de discours attendu (types et phases de la justification ${ }^{5}$ ) et c) au degré d'élaboration de la réflexion dans chaque épisode (jugé selon des critères de cohésion, pertinence, justesse et solidité du propos). Nous avons ensuite effectué des analyses quantitatives (statistiques descriptives avec SPSS et N'VIVO) afin de comparer les différents cas et classes (primaire/secondaire) pour juger d'une éventuelle progression entre les deux temps de mesure et d'éventuels effets de l'enseignement explicite de l'oral réflexif.

Pour nous aider à mieux comprendre les différences entre chaque élève-cas et les perceptions des enseignantes, nous avons d'autre part retranscrit et analysé le contenu des entretiens et bilans, puis analysé leur contenu par thèmes. Nous avions aussi consigné l'ensemble les données relatives aux différents outils de collecte pour pouvoir mieux contextualiser, interpréter et comparer les cas (voir extrait figure 2).

\footnotetext{
${ }^{4}$ Les types de reformulations : expressions de reformulation, auto-reformulations (répéter, expliciter, rectifier et synthétiser) et hétéro-reformulations (répéter, expliciter, rectifier et synthétiser).

${ }^{5}$ Les types de justifications : comparaisons, déductions/hypothèses, faits, opinions, savoirs littéraires, vécu.
} 


\begin{tabular}{|c|c|c|c|}
\hline & $\begin{array}{c}\text { Primaire }\left(6^{\circ}, \mathrm{MTL}\right) \\
\text { NL2-CL2 }\end{array}$ & Primaire $\left(6^{\circ}, \mathrm{MTL}\right)$ & Primaire $\left(6^{\circ}, M t l\right)$ \\
\hline $\begin{array}{l}\text { Éléments/ } \\
\text { Outils }\end{array}$ & $\begin{array}{l}\text { Cas 1-Fort (Emma) } \\
\text { responsable du temps préEex } \\
\text { Secrétaire Ex }\end{array}$ & $\begin{array}{l}\text { Cas 2-Moyen (Fabio) } \\
\text { secrétaire en préExp } \\
\text { responsable équipement Ex }\end{array}$ & Cas 3-Faible (Mélina) \\
\hline \multicolumn{4}{|c|}{ PRÉEXXPÉRIMENTATION } \\
\hline $\begin{array}{l}\text { Résultats scolaires } L \text { - } \\
E=0\end{array}$ & Élève forte et bonne lectrice & $\begin{array}{l}\text { Très fort en maths, pas intéressé et } \\
\text { beaucoup plus faible en lecture, agité, } \\
\text { dérange dans les } \mathrm{CL} \text {, vocabulaire limité } \\
\text { (Chinois d'origine) }\end{array}$ & $\begin{array}{l}\text { Faible en lecture, moyenne en } \\
\text { écriture et en oral, motivée par le } \\
\text { français, maîtrise mal le français } \\
\text { (Péruvienne au Québec depuis } 3 \\
\text { ans) }\end{array}$ \\
\hline $\begin{array}{l}\text { Autoévaluation } 1 \\
\text { (AE1) disc.: }\end{array}$ & En moyenne, elle se donne B. & En moyenne, il se donne B. & En moyenne, elle se donne B. \\
\hline $\begin{array}{l}\text { Autoévaluation } 2 \\
\text { (AE2) disc. }\end{array}$ & En moyenne, elle se donne B. & En moyenne, il se donne B. & En moyenne, elle se donne $B$. \\
\hline $\begin{array}{l}\text { Journal de lecture } \\
\text { (JL): }\end{array}$ & $\begin{array}{l}\text { Les commentaires de l'enseignante } \\
\text { sont positifs et encourageants. }\end{array}$ & $\begin{array}{l}\text { L'enseignante reprend par deux fois les } \\
\text { hypothèses et elle demande de faire des } \\
\text { commentaires plus pertinents. L'élève se } \\
\text { reprend à la } 4^{\bullet} \text { question/hypothèse. }\end{array}$ & $\begin{array}{l}\text { L'enseignante lui demande souvent } \\
\text { de mieux situer le moment de } \\
\text { l'histoire ou pourquoi tel passage } \\
\text { provoque la réaction chez l'élève. } \\
\text { Plusieurs félicitations pour les } \\
\text { stratégies. }\end{array}$ \\
\hline \multicolumn{4}{|c|}{ EXPÉRIMENTATION AVANT } \\
\hline $\begin{array}{l}\text { Portfolio (PF): } \\
\text { production initiale }\end{array}$ & $\begin{array}{l}\text { Ses forces: Écoute et respect du } \\
\text { sujet } \\
\text { Ses faiblesses : développement de } \\
\text { ses opinions, participation }\end{array}$ & $\begin{array}{l}\text { Ses forces : participation, écoute et respect } \\
\text { du sujet } \\
\text { Ses faiblesses : participation }\end{array}$ & $\begin{array}{l}\text { Ses forces : écoute, participation, } \\
\text { respect des autres } \\
\text { Ses faiblesses: description des } \\
\text { commentaires et des opinions }\end{array}$ \\
\hline \multicolumn{4}{|c|}{ EXPÉRIMENTATION PENDANT } \\
\hline $\begin{array}{l}\text { Portfollo (PF): } \\
\text { Ateller 1, fiche } \\
\text { synthèse } \\
\text { reformulation } \\
\text { (compréhension, } \\
\text { strotégies, } \\
\text { expressions) }\end{array}$ & $\begin{array}{l}\text { 1) a appris des expressions pour } \\
\text { reformuler et de nouvelles } \\
\text { stratégies. 2) s'est améliorée dans } \\
\text { la compréhension des idées des } \\
\text { autres. 3) utilisera les expressions, } \\
\text { se fera des films dans sa tête avant } \\
\text { de reformuler et utilisera les mots } \\
\text { importants. }\end{array}$ & $\begin{array}{l}\text { 1) a appris à mieux reformuler quand on } \\
\text { parle à quelqu'un. 2) rien. 3) rien. }\end{array}$ & $\begin{array}{l}\text { 1) a appris les stratégies et les } \\
\text { expressions pour mieux } \\
\text { reformuler. 2) rien 3) mettra en } \\
\text { pratique des stratégies pour } \\
\text { reformuler. }\end{array}$ \\
\hline Journal de lecture (J) & Note finale $B+$ & Note finale $\mathrm{D}+$ & Note finale B \\
\hline
\end{tabular}

Figure 2. Exemple de consignation des données pour trois élèves-cas

\section{4- Résultats et discussion}

Nous rapporterons les résultats pour chacun des trois indicateurs à l'étude (reformulation, justification et degré d'élaboration) en plus de commenter le degré de participation des élèves à la tâche et l'impression des enseignants sur l'ensemble de cette démarche. Pour ce faire, nous croiserons chaque fois les résultats quantitatifs, issus des analyses de contenu des transcriptions de CLP, et les résultats qualitatifs, issus des analyses de contenu des entretiens avec les élèves et les enseignants. Par ailleurs, comme de telles études de cas requièrent de citer les propos des participants, pour éviter les redondances, nous avons choisi de présenter de manière intégrée la section des résultats et celle de la discussion qui sont, normalement, deux sections distinctes.

\subsection{Différences observées dans l'utilisation des procédés de reformulation}

4.1.1 L'utilisation de la reformulation en général : différences et apports. Dans le cadre de cette recherche, l'analyse de la reformulation a porté sur neuf codes répartis en 
trois catégories (les expressions de reformulation, l'auto-reformulation et l'hétéroreformulation). Les résultats globaux montrent que toutes les équipes utilisent davantage la reformulation lors de l'expérimentation (passant d'une moyenne de 0,30 reformulation/minute à 0,80 ) et il en est de même pour tous les élèves-cas étudiés (passant d'une moyenne de 0,28 à 0,82 reformulation/minute de $\mathrm{CLP}^{6}$ ), et ce, peu importe leur type (fort, moyen, en difficulté) ou leur niveau scolaire (primaire, secondaire). Plus précisément, les élèves du primaire produisent deux fois plus de reformulations (de 0,30 à 0,69 reformulation/minute de CLP) et ceux du secondaire, trois fois plus (de 0,28 à 0,87 reformulation/minute de CLP). Bien que tous les types d'élèves progressent, ce sont les élèves forts qui réalisent la progression la plus importante. Cependant, la majorité des élèves-cas interrogés perçoivent ces progrès. Ainsi, 14 élèvescas sur 18 (dont cinq élèves forts, cinq moyens et quatre en difficulté) affirment, lors des entretiens, avoir fait des progrès en reformulation.

Plusieurs élèves affirment aussi qu'apprendre à reformuler les a aidés lors des cercles de lecture. Leur participation et leur compréhension ont été améliorées. Par exemple, Caroline ${ }^{7}(\mathrm{M}-\mathrm{Sec})^{8}$ affirme que la reformulation l'a aidée à être mieux comprise : ils [ses coéquipiers] me comprenaient plus pis j'avais moins de misère à tout dire. Pour Annabelle (D-Prim), la reformulation l'a surtout aidée à mieux comprendre les autres: des fois, il y avait des personnes qui utilisent pas les bons termes, les bonnes choses. Des fois je comprends pas qu'est-ce qu'ils veulent dire d'un côté, pis je leur ai demandé de reformuler et pis ils l'ont fait. Quant à Émeline (F-Prim), cela lui a donné des outils pour oser demander des clarifications : c'est que avant j'étais gênée. Je disais que je comprenais, mais en fait j'étais pas sûre de bien comprendre. Sauf que là, je me suis dégênée, avec cet atelier-là ça m'a plus aidée aussi. Enfin, pour Sherman (D-Sec), un élève dont le français n'est pas la langue maternelle, la reformulation pouvait faire la différence entre une incompréhension et un apprentissage : quand ils [ses coéquipiers] reformulaient j'apprenais mieux. [...] [Q]uand on questionnait et quand on disait nos stratégies, la plupart de notre équipe reformulait pas, mais Zac reformulait. Ça m'aidait mieux.

4.1.2 Les différents types de reformulation: des outils pour l'écoute, la compréhension et l'entraide. En ce qui concerne les types de reformulations utilisés, les résultats globaux montrent que tous les élèves ont recours à une plus grande variété, passant de huit à neuf types de reformulations lors de l'expérimentation. Cette progression est davantage marquée chez les élèves du secondaire que ceux du primaire, sans toutefois être statistiquement significative. Tous les élèves utilisent aussi plus souvent des reformulations plus complexes. En effet, l'emploi de reformulations qui permettent de construire la compréhension et de faire avancer les discussions, comme reformuler pour expliciter ou pour rectifier, augmente (respectivement de 0,09 à 0,17 et de 0,05 à 0,08 occurrence/minute de CLP). Au contraire, l'emploi de reformulations qui permettent de répéter des propos reste stable. L'utilisation d'expressions pour reformuler

\footnotetext{
${ }^{6}$ Cette différence est statistiquement significative (Wilcoxon signed rank $Z=-0,35 \mathrm{p}<0,005$ ).

${ }^{7}$ Tous les prénoms des participants ont été changés afin de respecter leur anonymat.

${ }^{8}$ Il faut comprendre ce code de la façon suivante : F signifie « fort », M signifie « moyen », D signifie « en difficulté »; Prim signifie « primaire » et Sec signifie « secondaire ».
} 
(par exemple : «si j'ai bien compris... ») est aussi en progression (passant de 0,01 à 0,05 occurrence/ minute de CLP). En ce qui concerne les élèves-cas, ils se comportent de façon similaire à l'ensemble des élèves : les élèves forts passent de sept à neuf types, les moyens de huit à neuf types et ceux en difficulté passent de six à huit types. Ce sont les élèves en difficulté du secondaire qui progressent le plus : ils doublent la variété des reformulations utilisées, passant de quatre à huit types différents.

De plus, les hétéro-reformulations, c'est-à-dire celles utilisées pour reformuler les propos d'autrui, progressent davantage que les auto-reformulations, utilisées pour reformuler ses propres propos. Cela est intéressant, car les hétéro-reformulations sont des indicateurs d'interactions entre les élèves. Par exemple, Caroline (M-Sec) précise quelques avantages qu'elle a perçus à ce sujet : [j'ai] pris plus de temps pour savoir de quoi je parlais et de ces affaires-là. Les choses que je disais aux autres pour qu'ils comprennent mieux. Je reformulais pour eux. Certains élèves plus forts parviennent à distinguer l'auto-reformulation de l'hétéro-reformulation, comme Laeticia (F-Prim) qui explique la reformulation en ces termes: [C'est] quand mettons tu expliques, que j'explique quelque chose pis que quand la personne a pas compris ça veut dire qu'il faut que tu redises dans des mots plus clairs qu'est-ce que t'as dit. Ou ça peut être le contraire, que si tu as mal compris que tu le redises dans tes mots à la personne pour voir c'est quoi que toi t'as compris, pour voir si tu as bien compris ce qu'on t'a expliqué.

Pour certains élèves, la reformulation est également liée à une meilleure écoute et à une participation plus active. Jessy (M-Prim), par exemple, affirme : il faut bien écouter et il faut bien comprendre parce que sinon on peut pas bien reformuler. [...] j'écoute mieux les autres parce que je veux savoir de quoi ils parlent et je veux aussi approfondir le sujet après. Pour d'autres, la reformulation est perçue comme un outil d'entraide entre pairs, comme l'explique Sabine (M-Prim): parce que mettons que les autres comprenaient pas j'essayais de l'expliquer en d'autres mots plus comprenables [sic]; ou comme le précise Annabelle (D-Prim): Quand on comprenait pas on demandait s'il pouvait reformuler d'une manière pour qu'on comprenne mieux.

Les enseignants engagés dans ce projet perçoivent également des changements à plus long terme à la suite des apprentissages réalisés en reformulation, notamment leur transfert dans d'autres domaines. Lors de l'entretien de suivi à la fin de l'année scolaire, Louis, enseignant du secondaire, affirme à ce sujet : Il y a des changements chez tous les élèves. Présentement quand ils se parlent, ils sont toujours à employer un nouveau mot à partir du projet. Par exemple : "Ah! J'ai fait une comparaison! Ah! J'ai reformulé! ».

En somme, les résultats montrent que tous les élèves utilisent de deux à trois fois plus les procédés de reformulation à la suite d'un atelier formatif à ce sujet. Bien que les élèves forts s'améliorent davantage, les élèves moyens et en difficulté démontrent également des progrès. Cependant, les apprentissages de ces derniers semblent moins assurés et il leur est plus difficile d'expliquer ce qu'est la reformulation et en quoi le fait d'utiliser de tels procédés a pu les aider dans les CLP. Toutefois, tous les élèves-cas ont démontré une meilleure habileté à utiliser des procédés de reformulation complexes qui sont, notamment, des indicateurs d'interactions entre les élèves, qui permettent de faire avancer les discussions et qui contribuent à une meilleure expression de la compréhension des sujets traités. La majorité des élèves-cas se disent conscients de ces 
progrès dans les entrevues et, selon les enseignants, ces apprentissages semblent avoir perduré au-delà du projet.

\subsection{Différences observées dans l'utilisation des procédés de justification}

4.2.1 L'utilisation d'éléments pour justifier son propos dans les CL : différences et apports. Les résultats globaux montrent que toutes les équipes utilisent davantage d'éléments pour justifier leurs propos lors de l'expérimentation (le nombre moyen de justifications passant de 0,37 à 0,49 justification/minute de $C_{L P}{ }^{9}$ ). En ce qui concerne les élèves-cas, seuls les élèves forts (qui passent de 0,39 à 0,65 justification/minute de CLP) et les moyens (qui passent de 0,40 à 0,66 ) font des progrès à ce sujet, alors que les élèves en difficulté justifient moins leurs propos que lors de la pré-expérimentation (passant de 0,29 à 0,19 justification/minute de CLP). Nous identifions deux causes possibles à cette contre-performance des élèves en difficulté : le fait que la capacité à justifier dépend des capacités en lecture et des connaissances acquises en littérature (ce qui a été très peu travaillé explicitement dans cette expérimentation) et le niveau de difficulté plus élevé du roman imposé à tous lors de $1^{\text {'expérimentation }}{ }^{10}$.

Au primaire, les élèves utilisent une proportion semblable de justifications entre la pré-expérimentation et l'expérimentation ( 0,40 et 0,44 justification/min de CLP). Quant aux élèves du secondaire, qui étaient à ce sujet plus faibles que les élèves du primaire au départ, ils améliorent davantage leur utilisation de justifications (passant de 0,33 à 0,56 justification/minute de CLP). Lors des entretiens, la moitié des élèves-cas ( 9 sur 18) affirment avoir fait des progrès en justification (dont deux élèves forts, quatre moyens et trois en difficulté). De plus, trois des six élèves-cas en difficulté sont d'avis que les procédés de justification les ont aidés lors des CLP, dont Francis (D-Prim) qui affirme : Bien genre d'habitude [...] on dit ouais on a compris voilà on passe au suivant. Mais là on discute plus, on dit «si j'ai bien compris » ou alors «donc », on justifie plus. C'est aussi le cas de Junior (D-Sec) qui y voit également un avantage dans la vie courante : Ça m'a aidé vu qu'en même temps je sais plus ce que je dis, parce que je le dis dans mes mots. Puis je justifie en même temps ce que j'ai dit. [...] Mais avant je faisais juste dire " ha j'aime pas cette personne elle est comme ça, c'est tout». Un autre, Sherman (D$\mathrm{Sec}$ ), affirme avoir appris, mais moins qu'en ce qui concerne la reformulation: Reformuler ça m'a plus aidé, mais justifier moins. [...] Parce que [...] j'avais pas vraiment dit pourquoi j'ai pris ce commentaire, pourquoi j'ai pris ce lien ou cette stratégie. Quant aux élèves-cas en difficulté qui affirment ne pas avoir progressé en justification, leurs explications varient, allant de je trouve qu'il n'y a rien vraiment à apprendre sur un oral. Tu l'as ou tu l'as pas (Sylvain, D-Sec), à ne pas se souvenir avoir appris à justifier ou à ne s'en rappeler que de façon confuse.

\footnotetext{
${ }^{9}$ Cette différence est statistiquement significative (Wilcoxon signed rank $Z=-2,06 \mathrm{p}=0,04$ ).

${ }^{10}$ En pré-expérimentation, chaque enseignant avait la liberté de faire lire à ses élèves le roman de son choix. En expérimentation, toutes les classes ont dû lire Le Passeur, un roman de science-fiction de Loïs Lowry (268 pages) qui se déroule dans une société utopique. Mais celle-ci se révèle dystopique pour Jonas, le personnage principal, lorsqu'il est appelé, à 12 ans, à occuper le poste à la fois le plus honorifique et le plus solitaire de sa communauté.
} 
4.2.2 Les différents types de justification : différences et apports observés. En ce qui concerne les différents types de justification utilisés pour fonder leurs propos, les faits et les déductions/hypothèses sont les plus utilisés, suivis de la comparaison et de l'opinion. Le recours aux savoirs littéraires et les références à son vécu sont les types les moins utilisés. Ce sont les déductions/hypothèses qui augmentent le plus lors de l'expérimentation. Lors des entretiens, peu d'élèves-cas font explicitement référence aux types de justifications qu'ils ont appris. Parmi les exceptions se trouve Émeline (F-Prim) qui affirme avoir appris les faits, exemples, explications, comparaisons. Elle ajoute: Avant c'était pas solide mes justifications mais avec ça je me suis plus aidée. Je donnais plus des..., je pense que maintenant c'est plus clair quand je justifie.

Quant au fait de citer un extrait de texte à l'appui d'un nouveau sujet dans la phase d'ouverture, tous les élèves-cas le font davantage en expérimentation. À ce sujet, les élèves du secondaire sont plus performants: ils citent deux fois plus le texte en ouverture que les élèves du primaire, alors que l'inverse s'était produit en préexpérimentation $^{11}$. Stella (F-Sec) donne un exemple de l'importance de justifier en s'appuyant sur le texte : [...] à cause des ateliers sur la justification, ça $m$ 'a aidée à donner plus de raisons et à mieux situer mes propos. Un autre élève, Sasha (F-Sec), ajoute : j'ai appris [...] à mieux justifier ce que je dis en oral. Donner plus d'exemples aussi en citant le texte.

En ce qui concerne les enseignants, leur perception est généralement positive au sujet de la justification. C'est le cas de Katherine, enseignante du secondaire, qui explique lors de l'entretien mené à la fin juin: Mes élèves sont plus conscients de comment arriver à justifier, que ce soit à l'oral ou à l'écrit. Je sens une espèce d'engouement pour essayer de trouver des réponses à tout ce qui est littéraire. Le transfert de cet apprentissage dans les autres matières a notamment été observé par les enseignants du primaire. Par exemple, Nathalie souligne que la justification est aussi utile dans les autres matières, notamment pour justifier en maths. Elle a également observé que ses élèves ont réellement fait des progrès en compréhension et justification et elle prévoit qu'en lecture, la réussite sera plus élevée; ils utilisent les stratégies et justifient plus. Cela semble même se refléter dans les résultats à l'examen de fin d'année, comme le souligne Claire, enseignante au primaire : Je suis certaine que cela les a aidés pour l'examen du MELS [ministère de l'Éducation, du Loisir et du Sport], les réponses aux questions d'interprétation et de justification étaient plus complètes, plus longues et mieux justifiées que les années précédentes. Finalement, les enseignants font état d'un effet positif sur leur enseignement. À ce sujet, Katherine affirme : Bref, je pense que je me suis découvert à travers ça en tant qu'enseignante: j'aime beaucoup faire ce genre de rétroactions-là avec mes élèves sur les lectures que l'on fait, par exemple [...]. [J]e me suis découvert une certaine motivation surtout envers la lecture littéraire et comment essayer [...] de justifier une réponse, par exemple. Ça m'a beaucoup motivée parce que ça me permet aussi d'établir un lien avec les élèves.

En somme, les résultats montrent que les élèves en utilisent en moyenne une plus grande proportion de procédés de justification lors des CL de l'expérimentation. C'est le cas de tous les types d'élèves-cas, à l'exception des élèves en difficulté, ce que l'absence

${ }^{11}$ À la pré-expérimentation, les élèves du primaire citaient deux fois plus souvent le texte que les élèves du secondaire. 
d'enseignement explicite des notions littéraires en général et le niveau de difficulté du roman peuvent peut-être expliquer. De plus, les résultats montrent que les élèves du secondaire ont davantage tiré profit de cet enseignement que les élèves du primaire, ce que la complexité cognitive de la tâche peut peut-être expliquer. Les élèves semblent aussi conscients de la difficulté plus grande à justifier (aspect discursif) qu'à reformuler (aspect langagier) : ils disent avoir éprouvé de plus grandes difficultés à justifier qu'à reformuler et seuls la moitié des élèves-cas affirment avoir fait des progrès en justification. Or, malgré ce qu'affirment les élèves, les enseignants soulignent tous avoir observé un transfert des apprentissages. Selon eux, leurs élèves justifieraient mieux leurs réponses, tant dans d'autres matières scolaires que dans les examens de lecture de fin d'année, ce qui s'avère être un effet particulièrement positif du projet.

\subsection{Différences observées dans le degré d'élaboration}

En ce qui concerne le degré d'élaboration des propos dans les CLP, toutes les équipes sauf une ont mieux justifié leurs propos en expérimentation (de 3,30 à 5,75 éléments de contenu pertinents par épisode au primaire; de 3,24 à 4,31 au secondaire) et toutes sauf une ont aussi moins abandonné de sujets ( $43 \%$ de sujets abandonnés en préexpérimentation contre $16 \%$ en expérimentation). Cependant, on constate que les élèves du primaire élaborent mieux leurs commentaires, et ce, pour un roman plus difficile, qui n'était pas de leur niveau. En fait, les élèves ont essentiellement échangé au sujet de leur compréhension littérale d'évènements factuels du roman ( $80 \%$ des épisodes), au détriment des autres modes de lecture attendus en lecture littéraire (plus personnel, textuel ou critique) (Hébert, 2014a).

Pour ce qui est des élèves-cas, ce sont surtout les élèves forts et, dans une moindre mesure, les élèves moyens qui ont contribué à l'élaboration des propos et progressé entre les deux conditions, comme on peut le constater dans le tableau 1 ci-dessous. Les élèves en difficulté, avec un roman plus difficile, n'ont pas progressé, mais ont néanmoins participé à l'élaboration des propos. Comme le dit Damien (M-Prim), Ouais le livre a changé et Le Passeur était plus difficile à comprendre que celui d'avant. Ça m'a déstabilisé un peu. Néanmoins, il estime avoir participé davantage : Au début quand les autres parlaient [...] je gardais mes opinions, je disais rien. C'est sûr que les stratégies ont aidé. Fait que là je dis plus mes idées et tout. 
Tableau 1

Nombre moyen de points en élaboration par CL selon les types d'élèves-cas

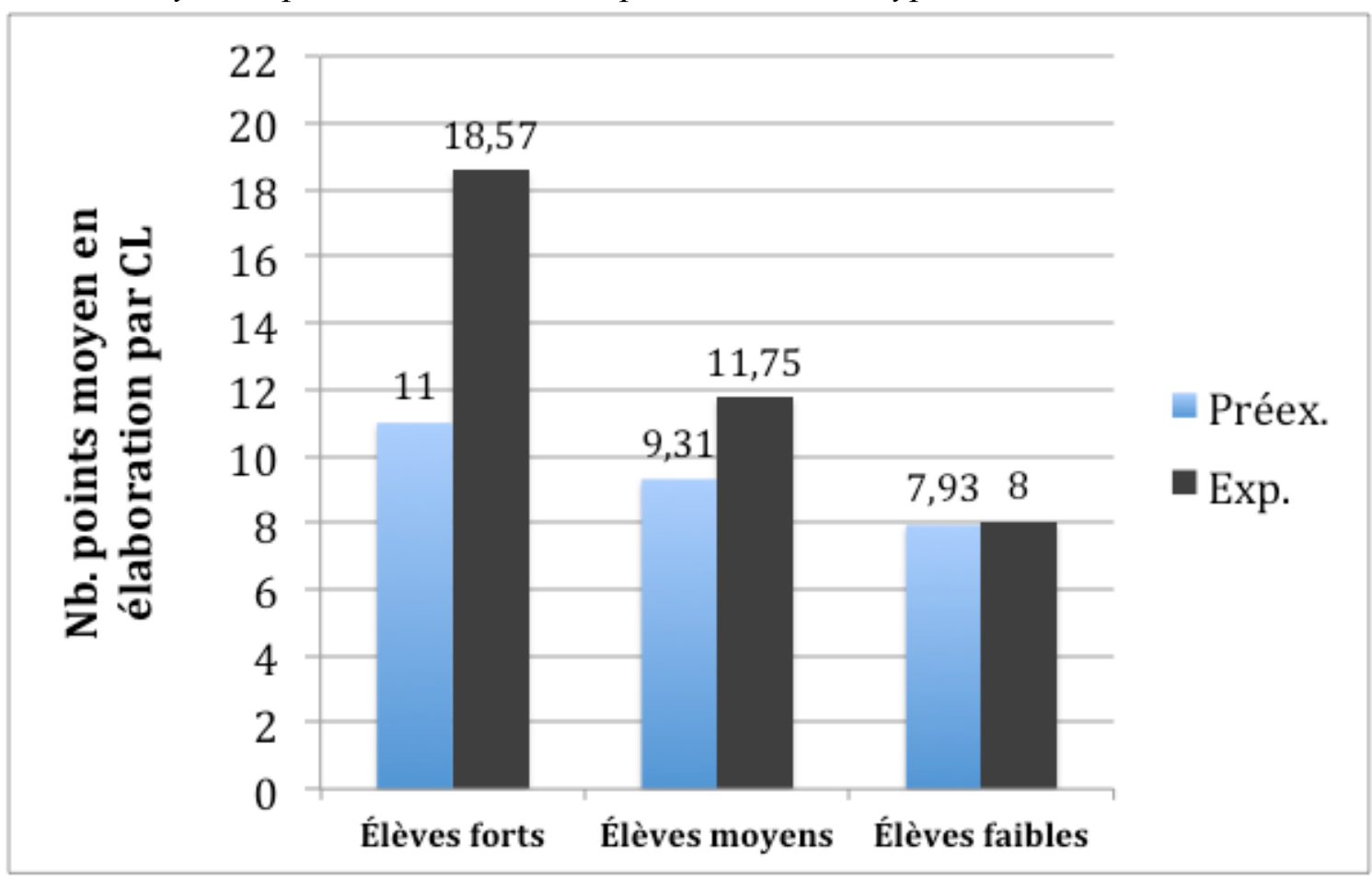

4.4 Différences observées dans l'engagement des élèves dans la tâche (équité, durée des échanges et degré de participation)

Entre les deux conditions, nous avons pu observer des améliorations quant à certains indicateurs qui peuvent traduire un meilleur engagement dans la tâche, comme la durée des échanges et l'équité des prises de parole. Cependant, nous ne pouvons pas affirmer que ces progrès sont des effets directs de l'enseignement de l'oral (selon les limites du design de cette recherche sans groupe contrôle), ils peuvent être attribuables à la simple maturation (reprise d'une même tâche quatre mois plus tard). Néanmoins, en expérimentation, les élèves font des CLP plus longs ${ }^{12}$; les différents épisodes ou sujets abordés le sont plus longuement ${ }^{13}$; puis les TP de chaque intervenant sont plus longs ${ }^{14}$. Enfin, la répartition des TP est aussi plus équitable à l'expérimentation ${ }^{15}$. Dans les entrevues, 10 élèves sur 18 disent également avoir mieux participé, et surtout les élèves moyens et en difficulté.

\footnotetext{
${ }^{12}$ Ils passent de 18 à 25 minutes au secondaire et de 13 à 21 minutes au primaire.

${ }^{13}$ Ils passent de 1,16 minutes à 2,11 minutes en moyenne sur les 172 épisodes analysés.

${ }^{14} \mathrm{Ils}$ passent de quatre à trois $\mathrm{TP} /$ minute au primaire et de cinq à quatre $\mathrm{TP} /$ minute au secondaire.

${ }^{15}$ La proportion des élèves participant de façon moyenne augmente de $30 \%$ à $49 \%$, alors que celle des élèves qui participent peu diminue de $37 \%$ à $27 \%$, comme celle des élèves qui participent beaucoup diminue de $33 \%$ à $24 \%$.
} 
En résumé, au-delà des résultats généraux qui montrent que les élèves ont reformulé et justifié davantage et de façon plus variée leurs propos, les entretiens avec les élèves-cas et les enseignants illustrent l'importance et les multiples fonctions de ces apprentissages en situation d'interaction entre pairs ainsi que l'intérêt de les enseigner explicitement. Et même si les élèves estiment avoir fait davantage de progrès dans l'utilisation de la reformulation que dans celle de la justification, c'est au sujet de la justification que les enseignants disent avoir davantage perçu une amélioration, par exemple lors des examens de fin d'année en lecture (au primaire) où les réponses leur semblaient plus élaborées que dans les années précédentes. Sans parler de la motivation et de la persévérance dans la tâche observées par la majorité des enseignants. Par exemple, Louis (sec), mentionne lors du bilan que les élèves ont participé à un degré inattendu. Beaucoup d'entre eux ont appris à utiliser des stratégies. De temps en temps, on entend un élève crier: Monsieur, je viens de reformuler ou de justifier, Monsieur, je viens de faires des liens. Il est aussi intéressant d'entendre des élèves dire : il faut se comporter comme dans un cercle de lecture. Je parle, tu m'écoutes et après on fera le contraire. Pour plusieurs enseignants, dont Nathalie (prim), il faut aussi attribuer ces progrès observés au fait d'avoir enseigné les compétences en intégration : C'est sûr que d'arrimer lecture, oral et écriture ça c'est gagnant aussi je trouve [...] c'est-à-dire que tout est pertinent pour les enfants [...], tout a été modelé avant. Donc quand on vient évaluer l'enfant, c'est clair qu'il avait tous les outils en main pour pouvoir faire le travail du mieux qu'il pouvait.

4.5 Intérêts et difficultés de cette approche intégrée pour le développement des habiletés en littératie

Les enseignants ont ainsi été souvent étonnés de l'engagement et de la persévérance de leurs élèves en lecture, étonnés de voir que la majorité des élèves ont voulu discuter avec sérieux de leurs valeurs et pu développer leur identité, leur autonomie interprétative et des attitudes plus positives face à la lecture. Les enseignants réalisent aussi que ces activités ouvertes et intégrées leur permettent de beaucoup mieux connaitre leurs élèves. Ils ont aussi mieux compris l'apport pour solidifier l'évaluation, et l'importance, pour l'apprentissage, de fournir un enseignement explicite de notions et stratégies de lecture littéraire et d'oral ciblées. Le rôle clé joué par les TIC pour l'enseignement-évaluation de l'oral et la motivation des élèves a aussi été observé (Hébert, 2014b). Ils ont été plusieurs aussi à remarquer un transfert des apprentissages (dans la capacité des élèves à mieux justifier dans l'examen du MELS, dans les échanges sociaux).

Cependant, parmi les difficultés éprouvées, ils ont constaté de sérieuses lacunes dans le degré de précision de leurs planifications annuelles (tant en appréciation des œuvres qu'en oral); puis des difficultés en ce qui a trait au choix de romans appropriés et à leur analyse pour mieux anticiper ce qui devrait être enseigné, et soutenir le questionnement des élèves plutôt que de poser des questions fermées. Ils ont trouvé difficile d'intégrer les compétences, d'avoir à gérer/évaluer un ensemble de tâches complexes s'étalant sur plusieurs semaines, plutôt que des leçons ponctuelles. Ils ont constaté par ailleurs n'avoir jamais ou presque enseigné l'oral (autrement que de faire faire des exposés) et avoué qu'il sera plus long de changer leurs pratiques dans ce cas. En 
général, en s'observant enseigner, ils ont mentionné ne pas assez interroger les connaissances antérieures des élèves; utiliser un métalangage spécialisé en littérature et soutenir une prise de notes efficace et à long terme; évaluer pour aider; puis faire de retours réflexifs constants sur les apprentissages en les identifiant clairement.

\section{5- Limites et conclusion}

L'étude visait à exposer les résultats quantitatifs obtenus en ce qui concerne la progression de trois indicateurs (reformulation, justification et degré d'élaboration), jugés importants dans un dispositif d'apprentissage entre pairs (CLP) faisant appel à l'oral dit réflexif, et ce, après enseignement explicite de l'oral. Sur un plan qualitatif, nous avons analysé et comparé les impressions de quelques élèves-cas et des enseignants à ce sujet. En résumé, au-delà des résultats généraux qui montrent que les élèves ont reformulé et justifié davantage et de façon plus variée leurs propos, les entretiens illustrent l'importance et les multiples fonctions socio-affective et cognitive de ces apprentissages en situation d'interaction entre pairs. Mais il faut rappeler qu'il s'agit d'une étude à visée descriptive et que rien ne peut être généralisé ici.

Il faut retenir de cette année d'expérimentation surtout l'effort d'intégration et de transposition en classe des deux cadres théoriques en didactique de la lecture et de l'oral, et ce, pour tenter d'analyser les indices de progression dans une activité où s'entrecroisent de nombreux enjeux sur le plan du développement des habiletés en littératie (compréhension/interprétation en lecture, puis aspects langagiers, discursifs et interactionnels en oral). L'enseignement de tous ces objets en intégration a constitué un défi important pour les enseignants, qui auraient grandement gagné à faire cette expérimentation sur deux années, le temps de s'approprier un seul modèle d'enseignement à la fois. Et si le choix d'avoir expérimenté ces modèles sans groupe contrôle se justifie à ce stade exploratoire de tentative d'intégration, il reste que de futures recherches devraient pouvoir mieux expliquer la cause des éléments de progression observés, notamment pouvoir mieux comprendre l'effet enseignant. Il faut aussi rappeler les nombreuses limites posées à l'interprétation de ces "progrès » en milieu naturel (le roman lu n'était pas le même dans les deux expérimentations; certaines équipes avaient changé; l'effet de désirabilité chez les élèves et les enseignants au moment de répondre aux questions dans les entretiens, dont certains ont été réalisés par la chercheuse qui avaient enseigné les ateliers en oral), puis continuer à questionner le fait que la majorité des enseignants aient avoué s'être rendu compte qu'ils n'enseignaient ni l'oral ni la littérature avant.

Malgré ces limites, nous croyons avoir réussi à atteindre notre objectif d'observer la faisabilité, et tout l'intérêt pour l'amélioration des échanges entre pairs, d'enseigner l'oral en intégration et de considérer l'oral réflexif à titre d'objet à enseigner explicitement. Ce type d'oral est encore complexe et difficile à cerner, mais nous parait tout à fait utile pour développer de manière intégrée l'ensemble des compétences en littératie qui sont nécessaires à la socialisation, à la construction des connaissances et à l'élaboration de la pensée en classe de français. 


\section{Références}

Berger, E. (2008). La reprise comme ressource interactionnelle en langue seconde. Travaux neuchâtelois de linguistique, 48, 4-61.

Bernié, J.-P. (2003). Fonction et fonctionnement de la discussion dans la construction de la classe comme communauté discursive. Communication présentée au Colloque La discussion en éducation et en formation, Montpellier : Université Paul-Valéry.

Blum, H. T., Lipsett L. R. \& Yocom D. J. (2002). Literature circles : A tool for selfdetermination in one middle school inclusive classroom. Remedial and Special Education, 23(2), 99-108.

Bouchard, R. (2004). «Apprentissage » de l'oral en L1 et pratiques de classe : un débat en $\mathrm{CP} / \mathrm{CE} 1$. Analyse interactionnelle et énonciative d'un dispositif innovant, le « petit laboratoire ». Dans A. Rabatel (dir.), Interactions orales en contexte didactique (p. 6789). Lyon, France : Presses universitaires de Lyon.

Cèbe, S., Goigoux, R. \& Thomazet, S. (2003). Enseigner la compréhension : principes didactiques, exemples de tâches et d'activités. « Enseigner le Français en Classes Relais », CD Rom édité par la prévention judiciaire de la jeunesse et la direction de l'enseignement scolaire, $38 \mathrm{p}$. http://dialogue.education.fr/D0049/lire_ecrire_enseigner_comprehension.pdf

Chabanne, J.-C. \& Bucheton D. (2002). Parler et écrire pour penser, apprendre et se construire. L'écrit et l'oral réflexif. Paris : Presses universitaires de France.

Chartrand, S.-G. \& Lord, M.-A. (2013). L'enseignement du français a peu changé depuis 25 ans. Québec français, 168, 86-89.

Crinon, J. (2002). Écrire le journal de ses apprentissages. Dans J.-C. Chabanne et D. Bucheton (dir.), Parler et écrire pour penser, apprendre et se construire. L'écrit et l'oral réflexifs (p. 123-143). Paris : Presses Universitaires de France.

De Pietro, J.-F. (1990). Approches linguistiques de l'interaction verbale et de son contexte. Tranel, 16, 11-36.

Deschênes, A.-J. (1991). Les stratégies de lecture. In Les entretiens Nathan sur la lectureActe 1, (p.29-45). Paris: Nathan.

Dolz, J. \& Schneuwly, B. (1997). Les genres scolaires, des pratiques langagières aux objets d'enseignement. Repères, 15, 27-41.

Dolz, J. \& Schneuwly B. (1998). Pour un enseignement de l'oral. Initiation aux genres formels à l'école. Paris : ESF éditeur.

Dreyfus, M. \& Cellier M. (2000). L'oral et la construction des savoirs dans des activités de français. Recherches, 33, 181-197.

Dumais, C. (2010). Atelier pour un enseignement de 1'oral. Québec français, 157, 58-59.

Fasel-Lauzon, V. (2009). L'explication dans les interactions en classe de langue: organisation des séquences, mobilisation de ressources, opportunités d'apprentissage. Thèse de doctorat inédite : Neuchâtel, Suisse : Université de Neuchâtel.

Fasel Lauzon, V., Pekarek-Doehler S. \& Pochon-Berger E. (2009). Identification et observabilité de la compétence d'interaction : le désaccord comme microcosme actionnel. Bulletin Suisse de linguistique appliquée, 89, 121-142.

Garcia-Debanc, C. \& Volteau S. (2007). Formes linguistiques et fonctions des reformulations dans les interactions scolaires. Recherches linguistiques (Usages et analyses de la reformulation), 29, 309-340. 
Hébert, M. (2003). Co-élaboration du sens dans les cercles littéraires entre pairs en première secondaire : étude des relations entre les modalités de lecture et de collaboration. Thèse de doctorat inédite, Université de Montréal, Montréal. En ligne à https://papyrus.bib.umontreal.ca/xmlui/handle/1866/6794

Hébert, M. (2004). « Pour une intégration des familles de situation en lecture littéraire ou les principes d'une approche transactionnelle. », Québec Français, 135, 82-84.

Hébert, M. (2006a). Une démarche intégrative pour enseigner à « apprécier des œuvres littéraires ». Québec Français, 143, 74-76.

Hébert, M. (2006b). Cahier pratique : exemple d'une séquence d'enseignement explicite pour 1'appréciation des œuvres. Québec Français, 143, 89-92.

Hébert, M. (2007). Oral et écrit réflexifs. Quelle interrelation pour le développement du commentaire? Dans G. Plessis-Bélair, L. Lafontaine \& R. Bergeron (dir.), La didactique du français oral au Québec (p. 92-117). Québec : Presses de l'Université du Québec.

Hébert, M. (2008). Le journal de lecture comme genre d'écrit réflexif, Québec Français, 149, 65-66.

Hébert, M. (2010). Littératie et école inclusive, un double défi pour l'école d'aujourd'hui. Dans M. Hébert et L. Lafontaine (dir.), Littératie et inclusion : outils et pratiques pédagogiques, Québec : Presses de l'Université du Québec, coll. Intervention, 1-10.

Hébert, M. \& L. Lafontaine (2012a). L'oral réflexif dans les cercles littéraires entre pairs : quelles caractéristiques cognitives, interactionnelles et discursives? Revue Lettrure, 2 , 116-128.

Hébert, M. \& Lafontaine, L. (2012b). L'oral réflexif dans les cercles littéraires entre pairs : analyse des conduites de justification en interaction, dans R. Bergeron et G.

Plessis-Bélair (dir.), Représentations, analyses et descriptions du français oral, de son utilisation et de son enseignement au primaire, au secondaire à l'université, Côte Saint-Luc : Éditions Peijac, 201-220.

Hébert, M. (2014a), Enseigner à reformuler dans les cercles de lecture entre pairs, Vivre le primaire, 27 (4), 47-49.

Hébert, M. (2014b), Les technologies au service de l'enseignement de l'oral dans les cercles de lecture entre pairs, Vivre le primaire, 27(4), 50-51.

Jacques, F. (1988). Étudier la situation interlocutive. Dans J. Cosnier, N. Gelas, N. KerbratOrecchioni et C. J. Kerbrat-Orecchioni (dir.), Échanges sur la conversation (p. 45-68). Lyon : CNRS.

Jaubert, M. (2005). Langage oral et construction de savoirs en classe de sciences. Communication présentée à la Journée d'étude Interactions langagières et apprentissages, Amiens, France.

Jaubert, M. \& Rebière, M. (2002). Parler et débattre pour apprendre: comment caractériser un « oral réflexif » ? Dans J.-C. Chabanne et D. Bucheton (dir.), Parler et écrire pour penser, apprendre et se construire. L'écrit et l'oral réflexifs (p. 163-186). Paris : Presses Universitaires de France.

Lafontaine, L. (2001). Élaboration d'un modèle didactique de la production orale en classe de français langue maternelle au secondaire. Thèse de doctorat inédite, Université du Québec à Montréal, Montréal. 
Lafontaine, L. \& Blain S. (2007). L’oral réflexif dans les groupes de révision rédactionnelle en quatrième année primaire au Québec et au Nouveau-Brunswick francophone. Dans G. Plessis-Bélair, L. Lafontaine et R. Bergeron (dir.), La didactique du français oral au Québec (p. 119-140). Québec : Les Presses de l'Université du Québec.

Lafontaine, L. \& Messier G. (2009). Les représentations de l'enseignement et de l'évaluation de l'oral chez des enseignants et des élèves du secondaire en français langue d'enseignement. Revue du Nouvel-Ontario, 34, p. 119-144.

Lafontaine, L \& Préfontaine, C. (2007). Modèle didactique de la production orale en classe de français langue première au secondaire. Revue des sciences de l'éducation, XXXIII(1), 47-66.

Le Cunff, C. (2004). Oral, savoirs, socialisation. Compétences langagières orales de la maternelle au collège. Rapport de recherche, IUFM de Bretagne, France.

Lusetti, M. (2004). Interactions verbales et gestion d'une tâche scolaire entre pairs. Dans A. Rabatel (dir.), Interactions orales en contexte didactique (p. 167-202). Lyon : Presses universitaires de Lyon.

McCraken, M. \& Murray, T. S. (2009). Stratégie nationale d'alphabétisation précoce. The economic benefits of literacy : Evidence and implications for public policy. London, Ontario : Réseau canadien de recherche sur le langage et l'alphabétisation. En ligne : http://www.dataangel.ca/docs/EconomicBenefitsofLiteracy2010.pdf

Ministère de l'Éducation, du Loisir et du Sport du Québec. (2007). Programme de formation de l'école québécoise. Français langue d'enseignement, secondaire $2^{e}$ cycle. Québec : Gouvernement du Québec.

Messier, G. (2007). Enseignement et apprentissage de l'oral en classe de français au secondaire : mise en œuvre d'un modèle didactique. Dans G. Plessis-Bélair, L. Lafontaine et R. Bergeron, La didactique du français oral au Québec (p. 45-62). Québec : Les Presses de 1'Université du Québec.

Ministère de l'Éducation du Québec. (2001). Programme de formation de l'école québécoise. Éducation préscolaire. Enseignement primaire. Québec : Gouvernement du Québec.

Nonnon, É. (1999). L'enseignement de l'oral et les interactions verbales en classe : champs de référence et problématiques. Revue française de pédagogie, 129, 87-131.

O'Brien, C. (2006). Investigation of the impact of video-based anchored instruction on the implementation of inclusive practices by students with learning disabilities (thèse de doctorat non publiée). Central Florida University, U.S.A.

Pekarek-Doehler, S., De Pietro, J.-F., Fasel Lauzon, V. \& Pochon-Berger, É. (2008). L'organisation du discours dans l'interaction en langue première et seconde: acquisition, enseignement, évaluation. Rapport final (projet CODI). Neuchâtel : Université de Neuchâtel.

PISA (2001). À la hauteur : La performance des jeunes du Canada en lecture, en mathématiques et en sciences (OCDE). Canada : Ministère de l'Industrie.

Sénéchal, K. (2012). La compréhension orale : la grande absente de la classe de français. Québec français, 164, 63-64.

Statistique Canada (2013). Les compétences au Canada: Premiers résultats du Programme pour l'évaluation internationale des compétences des adultes (PEICA). Canada: Ministère de l'Industrie. 


\section{Biographies des auteurs}

Manon Hébert est professeure agrégée au Département de didactique de l'Université de Montréal. Ses travaux portent sur la didactique de la lecture littéraire et visent à mieux comprendre le rôle de l'écrit et de l'oral réflexifs pour l'appréciation des œuvres littéraires, de même que les indices de progression de cette compétence sur un plan développemental (fin primaire et secondaire).

Amélie Guay est doctorante en éducation à l'Université du Québec en Outaouais et chargée de cours dans différentes universités québécoises. Ses travaux portent principalement sur l'apprentissage et l'enseignement du français (écriture, lecture et oral) au primaire, au secondaire et à l'université.

Lizanne Lafontaine est professeure titulaire à l'Université du Québec en Outaouais (UQO) et responsable de l'Équipe de recherche en littératie et inclusion (ÉRLI). Ses recherches portent sur la didactique de l'oral et la littératie, notamment en milieu défavorisé. Elle a publié divers ouvrages didactiques portant sur l'enseignement de l'oral au primaire et au secondaire et codirigé des ouvrages portant sur la didactique de l'oral et la littératie. Elle est également membre du Centre de recherche interuniversitaire sur la formation et la profession enseignante (CRIFPE).

\section{Annexe 1}

\section{Démarche d'enseignement et dispositifs}

\section{Phase de préexpérimentation (lecture, écriture) et précisions sur le dispositif des CLP:}

L'objectif des CLP est d'offrir à chaque élève l'occasion d'être soutenu pendant sa lecture. Chaque élève doit d'abord prendre en note sur des autocollants les stratégies de lecture qu'il a conscience d'utiliser au fur et à mesure de sa lecture (objectif métacognitif), puis en discuter en petit groupe et, ensuite, rédiger des commentaires écrits à ce propos dans son journal de lecture. Le déroulement des CLP est souple : de quatre à six élèves hétérogènes discutent pendant environ 30 minutes de stratégies de lecture choisies pendant leur lecture d'un même roman, et cela environ trois fois par roman. Chaque élève y joue un rôle précis (animateur, vérificateur, responsable de l'équipement, responsable du temps, secrétaire, rapporteur). Les deux rôles importants sont celui de l'animateur, qui s'assure que chaque élève prenne la parole pour présenter sa stratégie, et celui du secrétaire qui prend en note les stratégies discutées par chaque élève. Chaque prise de parole devrait être à peu près organisée ainsi (et selon le modèle attendu à l'écrit, dans le journal de lecture) : amener le sujet, en le situant dans l'histoire et en résumant le passage à discuter (citation à l'appui); puis poser son sujet (ici, cela signifie annoncer la stratégie de lecture utilisée); et justifier le propos en le «co-élaborant » entre pairs (Hébert, 2003).

\section{Phase d'expérimentation et étapes d'enseignement de l'oral réflexif:}

Les activités planifiées pour l'enseignement de l'oral réflexif intégré à cette situation de lecture se sont déroulées en trois phases : production initiale; les ateliers 
formatifs (3) et la production finale. En janvier, ces CLP filmés ont été réutilisés à titre de productions initiales. Toutes les séances d'enseignement ont été filmées et se sont étalées sur trois ou quatre semaines, à raison d'un atelier par semaine environ. Le modèle d'enseignement de l'oral développé par Lafontaine comporte trois grandes étapes : présentation de l'intention de communication générale (par exemple, lire un roman pour discuter et rédiger un journal de lecture); planification selon plusieurs paramètres (dont l'intégration des compétences) et réalisation d'une séquence didactique (production initiale; état des connaissances des élèves et retour sur leurs forces et faiblesses dans cette première production; ateliers d'enseignement explicite en oral, production finale et évaluation des notions enseignées) (voir exemple dans Hébert, 2014a). Ensuite, de mars à avril 2012, les élèves ont fait à nouveau trois CLP portant cette fois sur un même roman de science-fiction imposé à tous, puis des outils d'évaluation ont été développés et appliqués pour évaluer l'ensemble des activités en lecture (examen), en écriture (journal de lecture) et en oral (CLP).

a) Production initiale et état des connaissances des élèves. Pour entamer l'enseignement explicite de l'oral à l'hiver, les élèves ont d'abord rempli un portrait de locuteur, document dans lequel ils devaient se situer comme locuteur (situations scolaires dans lesquelles l'oral était une expérience positive ou négative). Par la suite, nous avons fait le retour sur la production initiale réalisée à l'automne, lors des CLP effectués pour la lecture du premier roman. Pour ce faire, les élèves ont d'abord écouté, à titre d'élément déclencheur et pour discuter de l'état de leurs connaissances, un document sonore démontrant des difficultés de communication entre deux personnes. Puis ils ont écouté une quinzaine de minutes de l'un de leurs CLP afin d'identifier dans leur portfolio trois forces et trois faiblesses de leur production orale d'équipe.

b) Ateliers formatifs. Nous rappelons que trois ateliers formatifs ont suivi et qu'ils étaient à considérer comme exploratoires sur le plan de la recherche. Les deux premiers (sur la reformulation et la justification) ont été préparés par les chercheuses et enseignés par Lafontaine; le troisième a été bâti par chaque équipe d'enseignants en collaboration avec leur conseillère pédagogique, et selon les forces et faiblesses identifiées dans leur classe respective. Chaque atelier respectait, en plus des critères de Lafontaine, les étapes proposées par Dumais (2010) : a) élément déclencheur, b) état des connaissances des élèves, c) enseignement, d) mise en pratique et e) retour en grand groupe.

c) Production finale. Pour l'évaluation, pendant la deuxième séries de CLP du printemps qui constituaient la " production finale », les enseignants circulaient pour observer les élèves à l'aide d'une grille d'observation qui rappelait les trois objets de l'oral enseignés lors des ateliers. Après chacun des CLP, les élèves réécoutaient leur CLP et remplissaient des grilles d'autoévaluation de groupe et individuelle portant sur les éléments enseignés. À la fin de l'expérimentation, les élèves ont également choisi leur meilleur CLP pour le soumettre à une évaluation finale qui a eu lieu en avril, à l'aide d'une grille de jugement final conçue par les chercheuses à partir des prescriptions ministérielles en vigueur. 


\section{1- À dire en classe devant tous les élèves :}

Bonjour, nous sommes rendues à la dernière étape de notre projet de recherche dans laquelle nous ferons des courtes entrevues à quelques élèves de la classe sur leurs apprentissages en oral. Ces élèves ont été sélectionnés au hasard parmi ceux qui ont signé le formulaire de consentement. Des garçons et des filles ont été sélectionnés dans cette pige au hasard. Nous vous rappelons qu'il était indiqué dans le formulaire que vous pourriez faire une entrevue en fin de projet.

\section{2- Entrevue : expliquer le but de l'entrevue aux élèves-cas}

Bonjour, on te rencontre parce qu'on voudrait savoir si tu as l'impression ou non d'avoir appris des choses en oral dans ce projet. Sens-toi bien à l'aise de répondre oui ou non. On va te poser ensuite quelques questions et si tu ne les comprends pas, tu peux nous le dire, on te les expliquera.

Questions : pour l'intervieweur, amener l'élève à en dire plus que ce qui est dans son portfolio. Par exemple, s'il lit simplement son portfolio lui demander s'il y a autre chose qu'il voudrait nous dire.

1- As-tu l'impression d'avoir appris quelque chose en oral au cours du projet? Pour répondre, quelle réponse cocherais-tu parmi celles-ci :

a) non, pas vraiment b) oui, un peu c) oui, moyennement b) oui, plusieurs choses a) oui, beaucoup de choses

2- Selon la réponse de l'élève, lui demander :

-Quoi, par exemple? (permettre de regarder dans le portfolio ou guider l'élève dans son portfolio s'il ne sait pas quoi répondre)

OU

Pourquoi dis-tu que tu n'as rien appris?

Sous-questions

i. Te souviens-tu d'avoir fait un atelier sur la reformulation? Qu'en retiens-tu? Est-ce que tu as l'impression que cela t'a aidé dans les $\mathrm{CL}$ ?

ii. Te souviens-tu d'avoir fait un atelier sur la justification? Qu'en retiens-tu? Est-ce que tu as l'impression que cela t'a aidé dans les CL?

iii. Atelier 3 libre

- CSDM : Tu as travaillé le mot DONC et la justification dans un atelier. As-tu appris quelque chose sur...? Qu'en retiens-tu? Est-ce que tu as l'impression que cela t'a aidé dans les $\mathrm{CL}$ ?

- CSMB : Tu as travaillé l'intonation et le volume dans un atelier. Qu'en retiens-tu? Est-ce que tu as l'impression que cela t'a aidé dans les $\mathrm{CL}$ ?

- CSRDN : Tu as travaillé sur la prise en compte des idées des autres dans le CL dans un atelier. Qu'en retiens-tu? Est-ce que tu as l'impression que cela t'a aidé dans les $\mathrm{CL}$ ?

- CSL : Tu as travaillé l'écoute active dans un atelier. Qu'en retiens-tu? Est-ce que tu as l'impression que cela t'a aidé dans les CL?

3. Tu viens de regarder ton portfolio à l'oral et de revoir ton portrait de locuteur (regarder le portrait avec l'élève). Qu'est-ce qui a changé dans ton portrait de locuteur? Pourquoi? Qu'est-ce qui n'a pas changé? Pourquoi?

Sous-question :

iv. Qu'as-tu coché dans ton portrait de locuteur qui est différent de la première version ? Pourquoi? Pourquoi ton portrait est resté pareil?

4. En priorité, sur quoi dois-tu encore travailler à l'oral pour mieux faire un CL?

a. Sous-question : Quelle(s) faiblesse(s) dois-tu encore travailler pour mieux faire un cercle de lecture?

5. Que voudrais-tu ajouter sur l'oral qui ne se trouve pas dans le portfolio? 